\title{
Update on laser-driven X-ray sources at ELI Beamlines
}

Jaroslav Nejdl, Uddhab Chaulagain, Ondrej Hort, Dong-Du Mai, Martin Albrecht, et al.

Jaroslav Nejdl, Uddhab Chaulagain, Ondrej Hort, Dong-Du Mai, Martin Albrecht, Matej Jurkovič, Ondřej Finke, Stefan Karatodorov, Marek Raclavský, Marcel Lamač, Eva Klimešová, Ziaul Hoque, Andreas Hult Roos, Maria Krikunova, Jakob Andreasson, "Update on laser-driven X-ray sources at ELI Beamlines," Proc. SPIE 11886, International Conference on X-Ray Lasers 2020, 1188608 (8 July 2021); doi: 10.1117/12.2593341

SPIE. Event: XVII International Conference on X-Ray Lasers, 2020, Online Only 


\title{
Update on laser-driven X-ray sources at ELI Beamlines
}

\author{
Jaroslav Nejdl*a,b, Uddhab Chaulagain ${ }^{\mathrm{a}}$, Ondrej Hort ${ }^{\mathrm{a}}$, Dong-Du Mai ${ }^{\mathrm{a}}$, Martin Albrecht ${ }^{\mathrm{a}, \mathrm{c}}$, Matej \\ Jurkoviča,c, Ondřej Finke ${ }^{\mathrm{a}, \mathrm{c}}$, Stefan Karatodorov ${ }^{\mathrm{a},+}$, Marek Raclavskýa,c, Marcel Lamača,d, Eva \\ Klimešováa, Ziaul Hoque ${ }^{\mathrm{a}}$, Andreas Hult Roos ${ }^{\mathrm{a}}$, Maria Krikunova ${ }^{\mathrm{a}}$, and Jakob Andreasson ${ }^{\mathrm{a}}$ \\ ${ }^{a}$ ELI Beamlines Center, Institute of Physics, Czech Academy of Sciences, Za Radnicí 835, Dolní \\ Břežany, Czech Republic; ' Institute of Plasma Physics, Za Slovankou 3, Prague, Czech Republic; \\ ${ }^{c}$ Faculty of Nuclear Sciences and Physical Engineering, Czech Technical University in Prague, \\ Břehová 7, Prague Czech Republic; dFaculty of Mathematics and Physics, Charles University, Ke \\ Karlovu 2, Prague, Czech Republic
}

\begin{abstract}
Three main paths have been selected within the ELI Beamlines research program for transforming driving laser pulses into brilliant beams of short wavelength radiation: High-order harmonic generation in gases, Plasma X-ray sources, and sources based on relativistic electron beams accelerated in laser-plasma. For each of these research areas, dedicated beamlines are built to provide a unique combination of X-ray sources to the user community. The employment of these beamlines has a well-defined balance between fundamental science and applications in different fields of science and technology. Besides those beamlines, a plasma betatron radiation source driven by the PW-class HAPLS laser system is being commissioned in the plasma physics platform to serve as a unique diagnostic tool for dense plasma and warm dense matter probing.
\end{abstract}

Keywords: X-rays, extreme ultraviolet, high-order harmonic generation, laser-plasma, plasma betatron, coherent diffraction imaging

\section{INTRODUCTION}

The mission of the ELI Beamlines center, which is a part of the pan-European ELI project, is to provide high energy beams of laser-driven X-ray sources and accelerated particles to the users from various fields of research [1]. In this paper, we provide a brief update on current and near-future experimental capabilities as well as some recent results on coherent diffraction imaging (CDI).

The main laser drivers considered for the X-ray sources are the kilohertz L1 Allegra laser system [2] and the multi-hertz petawatt-class L3 HAPLS system [3]. The L1 laser system is based on optical parametric chirped pulse amplification (OPCPA) and it is designed to provide pulse energy of $100 \mathrm{~mJ}$ with $15 \mathrm{fs}$ pulses at $1 \mathrm{kHz}$ repetition rate. This system already demonstrated operation above $50 \mathrm{~mJ}$ pulse energy, but the user operation is so far limited to $30 \mathrm{~mJ}$ with design values of pulse duration and repetition rate. The petawatt-class L3 laser is also still in the stage of ramping up its performance towards the design values. Currently, it provides pulses with the energy of $10 \mathrm{~J}$ and pulse length of $30 \mathrm{fs}$ at a maximum repetition rate of $3.3 \mathrm{~Hz}$ or in the single-shot mode. The petawatt operation is expected in near future, while the $10 \mathrm{~Hz}$ repetition rate will most likely become available in a few years.

\section{X-RAY SOURCES}

$\mathrm{X}$-ray sources that do not require very high energy of the driving laser pulse, such as high-order harmonic generation in gases or plasma X-ray sources, can be operated at a high repetition rate. In the case of the ELI Beamlines facility, those sources are driven by the kilohertz multi-terawatt L1 laser complemented by a commercial laser that provides $11 \mathrm{~mJ}$ pulses with duration of $35 \mathrm{fs}$ and repetition rate of $1 \mathrm{kHz}$. On the other hand, the sources based on laser acceleration of electrons to relativistic energies, such as plasma betatron and inverse Compton source, will be exclusively driven by the multi-hertz petawatt-class L3 laser.

*nejdl@fzu.cz; phone +420 266051 209; www.eli-beams.eu

+present affiliation: Institute of Solid State Physics Bulgarian Academy of Sciences, 72 Tsarigradsko Chaussee, Sofia 1784, Bulgaria

International Conference on X-Ray Lasers 2020, edited by Davide Bleiner,

Proc. of SPIE Vol. 11886, $1188608 \cdot$ C 2021 SPIE · CCC code:

0277-786X/21/\$21 - doi: $10.1117 / 12.2593341$ 


\subsection{HHG Beamline}

The HHG Beamline (see schematics in Fig. 1), as a source of coherent XUV radiation with femtosecond pulses based on high-order harmonic generation in noble gases that is driven by kilohertz laser drivers [4],[5], has been routinely employed for user experiments. Although the beamline is designed to accommodate up to $100 \mathrm{~mJ}$ laser pulses, the L1 laser currently provides a maximum pulse energy of $30 \mathrm{~mJ}$ for HHG. Laser focusing with f-numbers 80,200 , and 500 is currently utilized enabling efficient generation in various noble gases with both $\mathrm{kHz}$ laser drivers. The XUV beam is directed into two end-stations: a station dedicated to atomic molecular and optical sciences and coherent diffraction imaging [6] and a magneto-optical VUV ellipsometry station [7] dedicated to material science and surface science. Since the beamline commissioning various parts of it have been upgraded with respect to the original design to increase the photon number and improve the versatility and robustness of the source as well as to improve the characterization of the XUV beam [8].

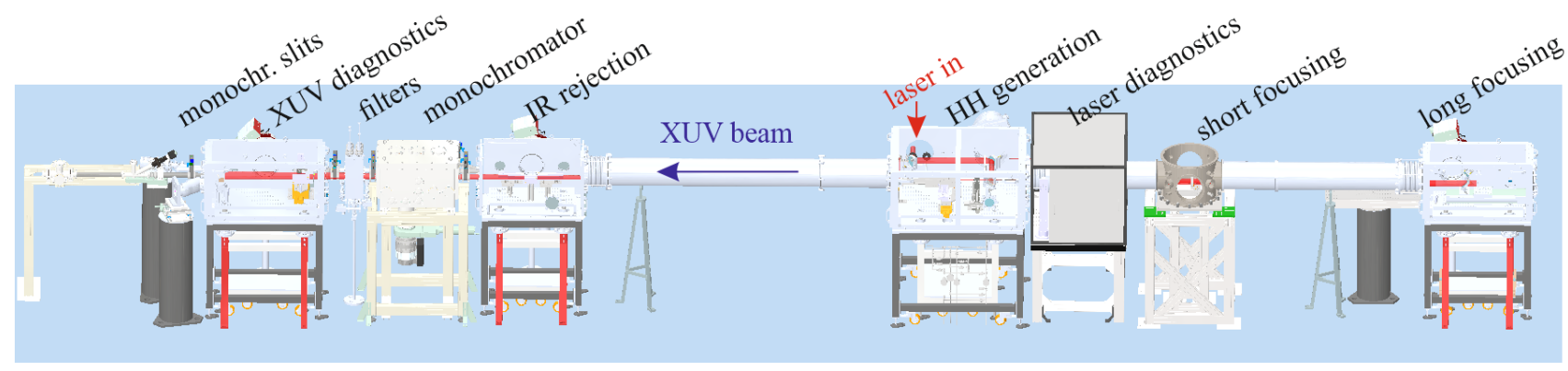

Figure 1. Drawing of the current state of the HHG Beamline with all vacuum chambers labeled by their main function. More details can be found in [4].

Recently, the grating monochromator employing a grating with 150 lines per $\mathrm{mm}$ was optimized to provide close to diffraction-limited image of the harmonic source on a panel with slits of various widths (50 to 400 microns) and 100, and $200 \mu \mathrm{m}$ pinholes. Fig. 2 shows a typical image from a Ce:YAG screen located at the position of the slit panel showing positions of $21^{\text {st }}$ and $19^{\text {th }}$ harmonics generated by the $792 \mathrm{~nm}$ Ti:Sapphire laser (11 mJ, $35 \mathrm{fs}, 1 \mathrm{kHz}$ ) in a Kr gas cell. The harmonics on the screen have around $200 \mu \mathrm{m}$ in full-width at half maximum.

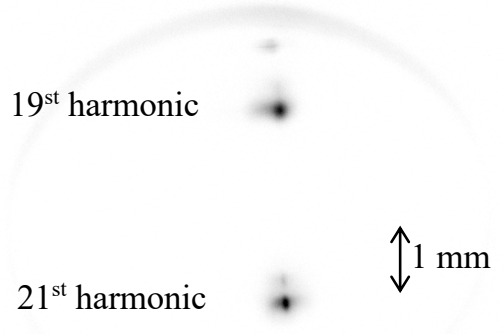

Figure 2. Negative image of the emission of the scintillator crystal that is placed in the position of exit slit of the monochromator showing $19^{\text {th }}$ and $21^{\text {st }}$ harmonic generated in krypton. The part of the circle at the top of the figure is caused by light coming from the edge of the scintillator.

In the following paragraph, we will describe a particular experiment, which proved the feasibility of using the beamline for monochromatic XUV coherent diffraction imaging. The monochromator slit or pinhole is imaged by an ellipsoidal mirror with demagnification factor of 5 onto a sample. The XUV focus was located $1 \mathrm{~mm}$ in front of the sample to ensure homogeneous illumination. A sample of $5 \mu \mathrm{m}$ by $4 \mu \mathrm{m}$ resembling the ELI logo was created by nano-structruring on $300 \mathrm{~nm}$ Au layer on a $75 \mathrm{~nm}$ thick $\mathrm{Si}_{3} \mathrm{~N}_{4}$ substrate using a focused ion beam. For the CDI experiment, the sample was illuminated by a monochromatic XUV beam of the 21 st harmonic at the wavelength of $38 \mathrm{~nm}$ (photon energy of $33 \mathrm{eV}$ ). Coherent diffraction patterns were captured using a back-illuminated CCD detector placed $50 \mathrm{~mm}$ behind the sample. Higher dynamic contrast of the pattern could be achieved utilizing a beam stop at $20 \mathrm{~mm}$ distance to the CCD sensor. 
Images with and without the central beam stop were acquired and processed in order to obtain a diffraction pattern containing both the diffraction center and good signal at higher Fourier frequencies (see Fig. 3). Even though a singleshot diffraction pattern could be recorded, a typical exposure time of the camera was $100 \mathrm{~ms}$ to acquire the lowest spatial frequency with a reasonable signal to noise ratio. To make the highest spatial frequency visible, an acquisition time of up to $15 \mathrm{~s}$ is needed, leading to an overexposure of the low-frequency features. The maximum spatial frequency recorded by such system was 7300 lines $/ \mathrm{mm}$ resulting in theoretical resolution of $\sim 90 \mathrm{~nm}$.
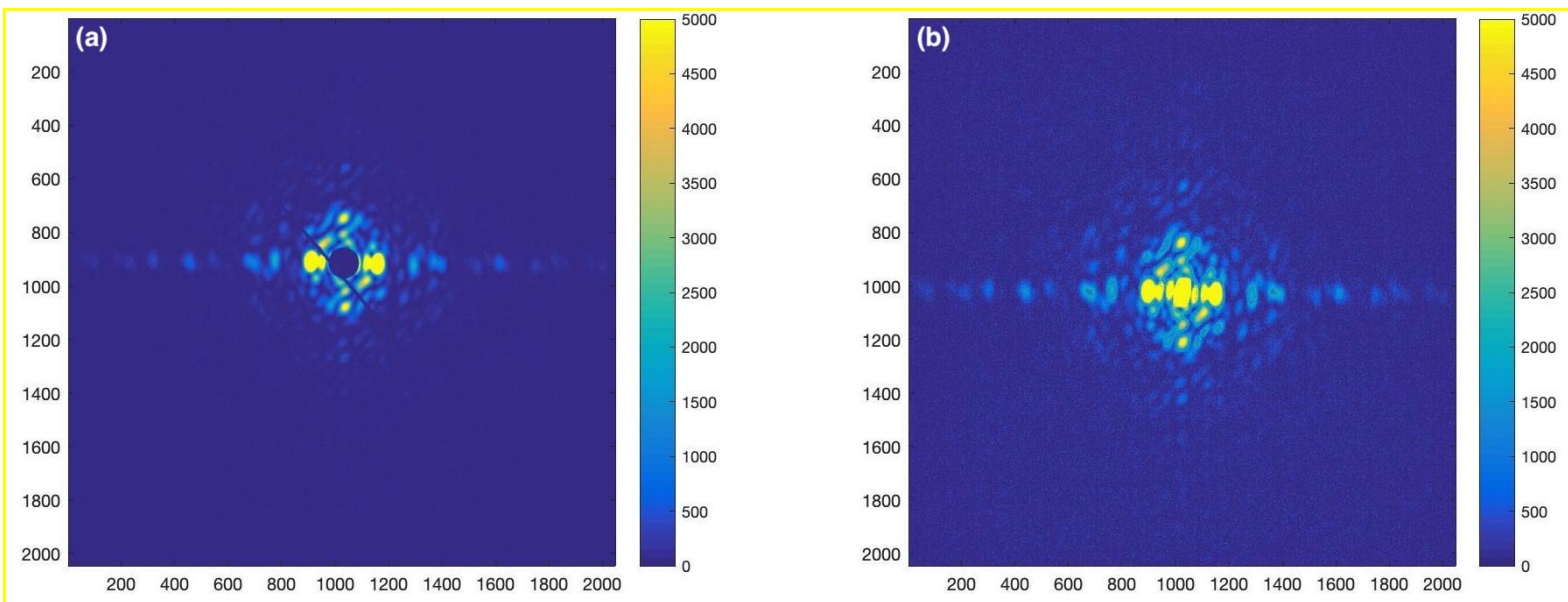

Figure 3. Raw diffraction pattern captured with beam stop (a), and post-processed, centered pattern (b) using a diffraction pattern without beam stop to reconstruct the parts of the pattern covered by the beam stop.

The applied phase retrieval algorithm is based on Fienup's Hybrid Input-Output [9][10] combined with Luke's RelaxedAveraged-Alternating-Reflections algorithm [11] to enhance the robustness to converge to the solution by fulfilling constraints in both Fourier and real space domains. Iterative reconstructions were performed following the strategy of incrementally optimized support masks, starting with a coarse auto-correlation envelope derived from the diffraction pattern itself. The support masks are optimized continuously by adopting the shape of the reconstructed object in real space. Optimized reconstructions are therefore achieved after 35 iterations pushing the image reconstruction to an almost real-time process. A qualitative comparison between the SEM image of the actual object and the reconstruction of its XUV image (see Fig. 4) reveals a good agreement both in scale and shape.
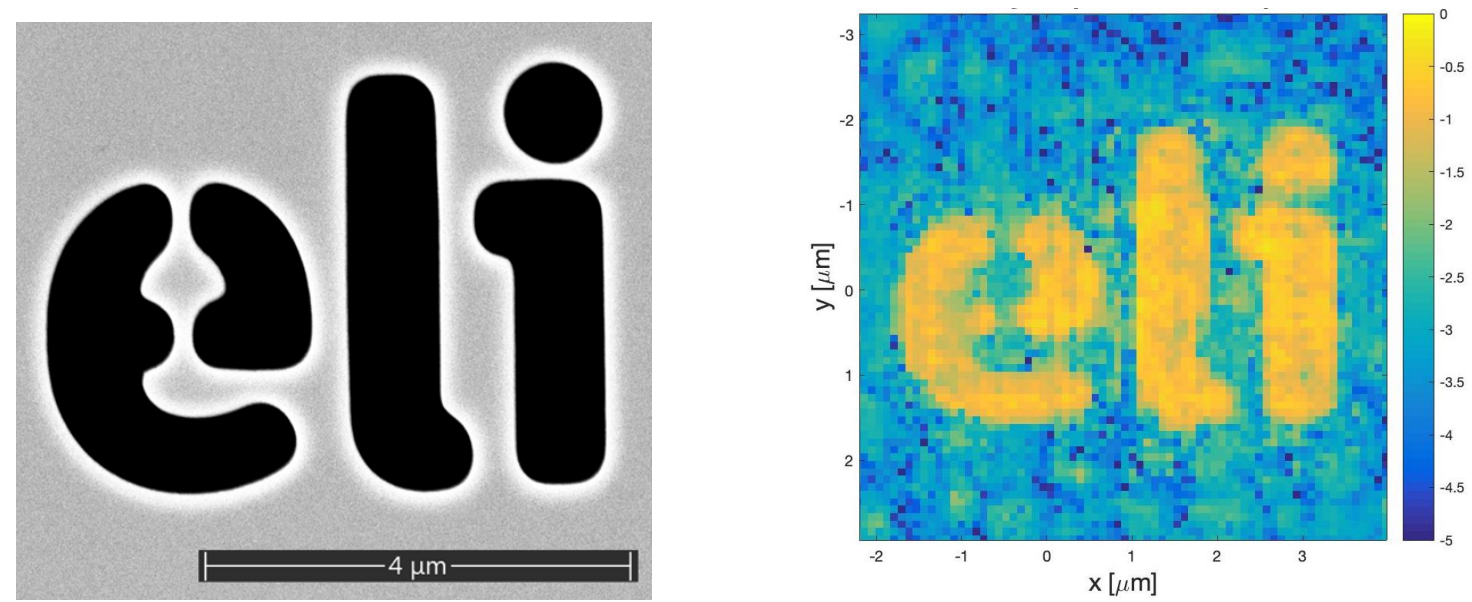

Figure 4. SEM image of ELI logo (left). Reconstructed image after 35 iterations (right). $21^{\text {st }}$ harmonic of a Ti:sapphire laser monochromatized by a grating monochromator was used for illumination. 


\subsection{Incoherent plasma X-ray sources}

There are two types of laser-plasma X-ray sources (PXS) depending on the state of the X-ray emitting material. Either it is the laser-generated plasma heated to high enough electron temperatures to generate appropriate ionization and collisional excitation of the working ion that consequently radiates through spontaneous emission, or it is the neutral target being bombarded by fast electrons from the hot plasma in its vicinity. The first type of source typically employs ns and ps lasers that are better absorbed and heat the plasma volume more efficiently resulting in ps-ns sources of XUV or soft X-ray radiation. The second type, on the other hand, employs femtosecond lasers that generate a significant population of hot electrons penetrating into the neutral material generating both a continuous X-ray spectrum (bremsstrahlung) and characteristic X-ray lines (such as $\mathrm{K}_{\alpha}$ or $\mathrm{L}_{\alpha}$ lines) via electron collisions with neutral atoms of the target. The later type of the source, if properly designed, is capable of providing sub-picosecond pulses of hard X-ray radiation (few keV $-30 \mathrm{keV}$ ) with reasonable efficiency. Therefore, this method has been chosen to be implemented at ELI Beamlines for ultrafast probing of structural and electron dynamics by diffraction methods and spectroscopy, respectively. Proper laser intensity sets a suitable electron temperature for generation in a given spectral range. The hot electron temperature scales as $\mathrm{T}_{\text {нот }} \sim\left(\mathrm{I} \lambda^{2}\right)^{1 / 3}$ underlining the importance of development of long-wavelength high-peak power laser systems. Moreover, a contrast of the laser is a very important aspect, as it defines the interaction conditions of the pulse with the target.

There are three types of high-density targets being investigated for laser-plasma X-ray sources with kilohertz laser drivers ( $\mathrm{L} 1$ and commercial $\mathrm{kHz}$ laser) focused by short focal-length parabolic mirror. A water jet target emitting lowenergy X-ray continuum [12], metal tape target $(\mathrm{Cu}$ and Mo tapes) with its characteristic lines and significant bremsstrahlung in the 10-20 keV energy range [13], and liquid metal jet source [14] using Ga or Bi/In as a renewable target. While the water jet source lacks characteristic lines and therefore offers low spectral brightness, its advantage lies in its almost debris-free operation, making it an easy-to-implement/operate X-ray source for spectroscopy applications. Sufficient photon flux generated by metal target PXS systems come with debris contamination of the reaction chamber, making the sources prone to operation instability in long-term runs and flux output. Thus, each of these sources has its advantages and disadvantages. Further investigation is needed to decide which technology will become the basis for user experiments.

\subsection{Laser-plasma accelerator-based X-ray sources}

The Gammatron beamline, a multidisciplinary user-oriented source of hard X-ray radiation based on the laser-plasma accelerator (LPA) is being deployed in experimental hall E2 of the ELI Beamlines. The Gammatron beamline [15] consists of a broadband hard $\mathrm{X}$-ray source with spectral range from a few $\mathrm{keV}$ to few hundreds of $\mathrm{keV}$ through a betatron scheme and a quasi-monoenergetic beam of hard X-rays with a spectral range of a few 100's keV in inverse Compton scheme. The mechanism of generation of these X-ray sources is based on laser wakefield electron acceleration, in which an intense $\left(>10^{18} \mathrm{~W} / \mathrm{cm}^{2}\right)$ femtosecond laser pulse interacts with a gas medium. The ponderomotive force of laser drives an electron plasma wave that traps electrons and accelerates them to relativistic energies. The accelerated charge also experiences transverse oscillations due to restoring force induced by space-charge separation, which generates synchrotron like radiation. As a result, a collimated X-ray beam with bright ultrashort pulses known as plasma betatron radiation is generated [16]-[17]. Recent experimental results on Betatron show that up to $10^{11}$ photons/shot can be routinely delivered [18]-[19], the photon flux can be further enhanced by using the PW class lasers. Furthermore, in the inverse Compton scheme, where the relativistic electrons collide with a counter-propagating intense laser pulse, gamma radiation (100's of $\mathrm{keV}$ to the $\mathrm{MeV}$ range) can be generated as a result of double Doppler upshift of the scattered laser pulse. Both of these X-ray sources can deliver ultrashort (few fs) X-ray or gamma-ray pulses as they inherit the temporal properties of the accelerated electron pulse. Those sources represent the emergence of a new generation of bright, collimated, compact X-ray sources.

The driving laser of the Gammatron beamlines is a state-of-the-art diode-pumped Ti:sapphire L3 HAPLS laser that should deliver up to $30 \mathrm{~J}$ at a $10 \mathrm{~Hz}$ repetition rate. The schematic of the Gammatron beamline is shown in Figure $5 \mathrm{~A}$. The target for the generation of relativistic electron bunches and X-rays will be either supersonic gas jet or gas cell. The typical gases for the LPA target are pure He, He with the admixture of Nitrogen, gas clusters, or dry air [20], and the plasma density for the acceleration will be in the order of $10^{18-19} \mathrm{~cm}^{-3}$. The gas jets are characterized using a high resolution, high -sensitivity interferometer [21]-[22] before being placed into the experimental chamber. The ultrashort pulses ( $\mathrm{fs})$ of Gammatron sources and their efficient synchronization with another femtosecond laser pulse offer a 
versatile tool in studying various time-resolved experiments in ultrafast science. The major applications of the Gammatron source include X-ray phase-contrast imaging [23], radiography, time-resolved X-ray absorption spectroscopy [24], X-ray diffraction, and industrial imaging of large and dense objects.
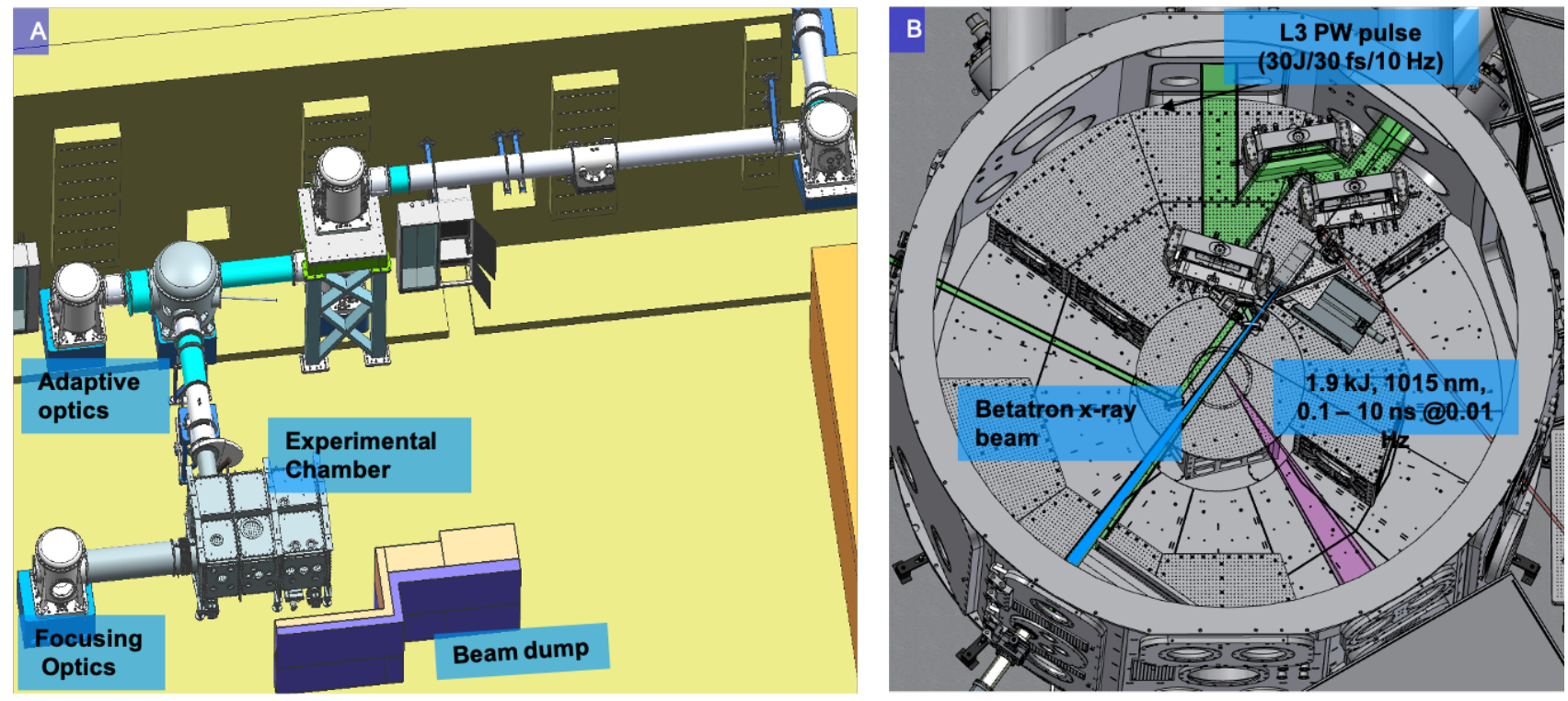

Figure 5 A) Schematic of Gammatron beamline in the E2 experimental hall at ELI Beamlines. B) Multi beams setup inside P3 chamber, a typical setup for plasma and WDM probing. The green rectangular beam is focused into a supersonic gas jet using a spherical mirror with a $5 \mathrm{~m}$ focal length to accelerate electrons and generate a betatron X-ray source (blue beam).

A separate betatron X-ray source [25] is being developed in the plasma physics platform (P3) [26] located in the experimental hall E3 (Fig. $5 \mathrm{~B}$ ). The P3 facility is focused on a range of plasma physics experiments including high energy density (HED) physics, ultra-intense laser-matter interaction, laboratory astrophysics, and advanced plasma physics studies using multiple laser and particle beams [27]. Dense and highly ionized plasma can be produced during the interaction of intense laser with matter. Probing such plasmas requires a very bright hard X-ray source to overcome the plasma self-emission and to penetrate through it. Furthermore, it will be an excellent probe for many laboratory astrophysics experiments like collisionless shock or radiative shock waves [28]-[30]. In those experiments, the object moves very fast, the shock front and hydrodynamic instabilities can be very thin, i.e. requiring an ultrashort probe to resolve their structure. In laser-heated warm dense matter (WDM), the transition effects happen on timescales of the order of few tens of femtoseconds [31]. Therefore, the probing source of the WDM needs to be extremely short. The Betatron X-ray source offers X-ray pulses with a duration of tens of fs, delivers a collimated beam with a divergence less than few tens of milliradians, and small source size making the source ideal backlighting tools to study time-resolved ultrafast processes in plasma physics experiments.

\section{CONCLUSION AND OUTLOOK}

A new generation of X-ray sources (see also Sect. 1 and Sect. 2 [32]), driven by high power laser systems, is increasingly available for user research projects at the ELI Beamlines center. The co-existence of primary and secondary photon sources operating at a variety of energies with both monochromatic/coherent and polychromatic/incoherent operation modes allow the synergetic usage of sources and beamlines. Thus, not only classical IR-pump/X-ray-probe experiments can be carried out but also XUV/X-ray pump/probe concepts will be feasible. Covering pump/probe energies from near IR, over XUV close to the water window, and up to hard X-rays in the $\mathrm{keV}$ regime, the sources at ELI Beamlines offer unique experimental opportunities for the international research community. In time, we expect that these sources will outperform similar-type sources available in small laboratories not only in terms of photon flux but also in robustness, stability, and availability of well-established user end-stations equipped with extraordinary sets of diagnostics. Moreover, further development of laser-driven X-ray sources will improve the usability of their compact versions that are more generally available in small university laboratories. 


\section{Acknowledgments}

This research was supported by the project Advanced research (ADONIS) (CZ.02.1.01/0.0/0.0/16_019/0000789) and by the project High Field Initiative (HiFI) (CZ.02.1.01/0.0/0.0/15_003/0000449), both from European Regional Development Fund. The results were obtained with the financial support of the Ministry of Education, Youth, and Sports as part of targeted support of Large infrastructures (project LM2015065) and the Grant Agency of the Czech Republic (project 18-27340S).

\section{REFERENCES}

[1] Rus, B. et al. "Outline of the ELI-Beamlines facility", Proc. SPIE 8080, 808010 (2011).

[2] Antipenkov, R. et al., "The construction of Allegra kilohertz femtosecond laser system at ELI-Beamlines," Proc. SPIE 11034, 110340M (2019).

[3] Sistrunk, T. et al. "All Diode-Pumped, High-repetition-rate Advanced Petawatt Laser System (HAPLS)," in Conference on Lasers and Electro-Optics, OSA Technical Digest (online) (Optical Society of America, 2017), paper STh1L.2.

[4] Hort, O. et al., "High-flux source of coherent XUV pulses for user applications," Opt. Express 27, 8871 (2019).

[5] Nejdl, J. et al., "Progress on laser-driven X-ray sources at ELI Beamlines", Proc. SPIE 11111, 11110I (2019).

[6] Klimešová, E. et al. "A Multipurpose End-Station for Atomic, Molecular and Optical Sciences and Coherent Diffractive Imaging at ELI Beamlines" submitted

[7] Espinoza, S. et al. "Characterization of the high harmonics source for the VUV ellipsometer at ELI Beamlines," Journal of Vacuum Science \& Technology B 38, 024005 (2020).

[8] Jurkovič, M. et al. (in preparation)

[9] Fienup, J. R. "Reconstruction of an object from the modulus of its Fourier transform," Optics Letters 3, 27 (1978).

[10] Luke, D. R. et al., "Phase retrieval, error reduction algorithm, and Fienup variants: A view from convex optimization," J. Opt. Soc. Am. A. 19, 1334 (2002).

[11] Luke, D. R. "Relaxed averaged alternating reflections for diffraction imaging," Inverse Problems 21, 37 (2005).

[12] Miaja-Avila, L. et al. "Laser plasma x-ray source for ultrafast time-resolved x-ray absorption spectroscopy," Structural Dynamics 2, 024301 (2015).

[13]Zamponi, F. et. al. "Femtosecond hard X-ray plasma sources with a kilohertz repetition rate," Applied Physics A 96, 51 (2009).

[14] Reich, Ch. et al. "Ultrafast x-ray pulses emitted from a liquid mercury laser target," Opt. Lett. 32, 427 (2007).

[15] Chaulagain, U., et al. "ELI Gammatron Beamline: Dawn of ultrafast hard X-ray science" (in preparation).

[16] Ta Phuoc, K., et al. "Demonstration of the ultrafast nature of laser-produced betatron radiation." Physics of Plasmas 14, 080701 (2007).

[17] Horný, V. et al." Temporal Profile of Betatron Radiation from Laser-Driven Electron Accelerators: Numerical Study", Phys. Plasmas 24, 063107 (2017).

[18] Kozlová, M. et al., "Hard X Rays from Laser-Wakefield Accelerators in Density Tailored Plasmas," Phys. Rev. X 10, 011061 (2020).

[19] Fourmaux, S. et al., "Laser-based synchrotron X-ray radiation experimental scaling.” Opt. Express 28, 3147 (2020).

[20] Boháček, K., et al. "Stable electron beams from laser wakefield acceleration with few-terawatt driver using a supersonic air jet." Nuclear Instruments and Methods in Physics Research Section A: Accelerators, Spectrometers, Detectors and Associated Equipment 883, 24 (2018).

[21] Nejdl, J. et al., "Imaging Michelson interferometer for a low-density gas jet characterization" Rev. Sci. Instr. 90, 065107 (2019).

[22] Karatodorov, S., et al., Multi-pass probing for high-sensitivity tomographic interferometry (submitted)

[23] Chaulagain, U., et al., "X-ray phase contrast imaging of biological samples using a betatron X-ray source generated in a laser wakefield accelerator" Proc. SPIE, 10240, 1024014 (2017). 
[24] Mahieu, B., et al., "Probing warm dense matter using femtosecond x-ray absorption spectroscopy with a laserproduced betatron source" Nat. commun. 9, 1 (2018).

[25] Chaulagain, U., et al. "LWFA-Driven Betatron Source for Plasma Physics Platform at ELI Beamlines." International Conference on X-ray Lasers. Springer, Cham, 2018.

[26] Weber, S. A. et al. "P3: An installation for high-energy density plasma physics and ultra-high intensity laser-matter interaction at ELI-Beamlines", MRE 2, 149 (2017).

[27] Jourdain, N., et al., "The L4n laser beamline of the P3-installation: Towards high-repetition rate high-energy density physics at ELI-Beamlines" Matter and Radiation at Extremes 6, 015401 (2020).

[28] Chaulagain, U. et al., "Structure of a laser-driven radiative shock", High Energy Density Phys. 17A, 106 (2015).

[29] Clayson, T., et al. "Counter-propagating radiative shock experiments on the Orion laser and the formation of radiative precursors." High Energy Density Physics 23, 60 (2017).

[30] Singh, R. L., et al. "Experimental study of the interaction of two laser-driven radiative shocks at the PALS laser." High Energy Density Physics 23, 20 (2017).

[31] Falk, K., "Experimental methods for warm dense matter research," High Power Laser Science and Engineering 6, E59 (2018).

[32] D. Bleiner, The Science and Technology of X-ray Lasers: A 2020 Update Proc. SPIE 11886-00 (2021) 